\title{
TEM Analyses of Itokawa Regolith Grains and Lunar Soil Grains to Directly Determine Space Weathering Rates on Airless Bodies
}

\author{
Eve L. Berger ${ }^{1}$, Lindsay P. Keller ${ }^{2}$ and Roy Christofferson ${ }^{3}$ \\ 1. Geocontrol Systems, Inc - Jacobs JETS contract - NASA Johnson Space Center, Houston USA \\ 2. NASA Johnson Space Center, Houston USA \\ 3. Jacobs Technology - NASA Johnson Space Center, Houston USA
}

Samples returned from the moon and Asteroid Itokawa by NASA's Apollo Missions and JAXA's Hayabusa Mission, respectively, provide a unique record of their interaction with the space environment. Space weathering effects result from micrometeorite impact activity and interactions with the solar wind. While the effects of solar wind interactions, ion implantation and solar flare particle track accumulation, have been studied extensively, the rate at which these effects accumulate in samples on airless bodies has not been conclusively determined. Results of numerical modeling and experimental simulations do not converge with observations from natural samples.

We measured track densities and rim thicknesses of three olivine grains from Itokawa and multiple olivine and anorthite grains from Lunar soils of varying exposure ages [1,2,3]. Samples were prepared for analysis using a Leica EM UC6 ultramicrotome and an FEI Quanta 3D dual beam focused ion beam-scanning electron microscope (FIB-SEM) [4]. Transmission electron microscope (TEM) analyses were performed on the JEOL 2500SE 200kV field emission STEM.

The solar wind damaged rims on Lunar anorthite grains are amorphous, lack inclusions, and are compositionally similar to the host grain. The rim width increases as a smooth function of exposure age until it levels off at $\sim 180 \mathrm{~nm}$ after $\sim 20$ My (Fig. 1). While solar wind ion damage can only accumulate while the grain is in a direct line of sight to the Sun, solar flare particles can penetrate to mm-depths. To assess whether the track density accurately predicts surface exposure, we measured the rim width and track density in olivine and anorthite from the surface of rock 64455 [5], which was never buried and has a surface exposure age of $2 \mathrm{My}$ based on isotopic measurements [6]. The rim width from 64455 (60-70nm) plots within error of the well-defined trend for solar wind amorphized rims in Fig. 1. Measured solar flare track densities are accurately reflecting the surface exposure of the grains. Track densities correlate with the amorphous rim thicknesses.

While the space-weathered rims of anorthite grains are amorphous, the space-weathered rims on both Itokawa and Lunar olivine grains show solar wind damaged rims that are not amorphous. Instead, the rims are nanocrystalline with high dislocation densities and sparse inclusions of nanophase Fe metal $[2,5,7,8]$. The rim thicknesses on the olivine grains also correlate with track density. The Itokawa olivine grains have track densities that indicate surface exposures of $\sim 10^{5}$ years [9]. Longer exposures (up to $\sim 10^{7}$ years) do not amorphize the rims, as evidenced by lunar soil olivines with high track densities $\left(\sim 10^{11} \mathrm{~cm}^{-2}\right)$. From the combined data, shown in Fig. 1, it is clear that olivine is damaged (but not amorphized) more rapidly by the solar wind compared to anorthite. The olivine damaged rim forms quickly (in $\sim 10^{6} \mathrm{y}$ ) and saturates at $\sim 120 \mathrm{~nm}$ with longer exposure time.

The anorthite damaged rims form more slowly, amorphize, and grow thicker than the olivine rims. This is in agreement with numerical modeling data which predicts that solar wind damaged rims on anorthite will be thicker than olivine $[10,11]$. However, the models predict that both olivine and anorthite rims will 
amorphize and reach equilibrium widths in $<10^{3} \mathrm{y}$, in contrast to what is observed for natural samples. Laboratory irradiation experiments, which show rapid formation of fully amorphous and blistered surfaces from simulated solar wind exposures [e.g. 10,12] are also in contrast to observations of natural samples. These results suggest that there is a flux dependence on the type and extent of irradiation damage that develops in olivine. This flux dependence suggests that great caution be used in extrapolating between high-flux laboratory experiments and the natural case, as demonstrated by [12].

We constrain the space weathering rate through analysis of returned samples. Provided that the track densities and the solar wind damaged rim widths exhibited by the Itokawa grains are typical of the finegrained regions of Itokawa, then the space weathering rate is on the order of $10^{5} \mathrm{y}$. Space weathering effects in lunar soils saturate within a few My of exposure while those in Itokawa regolith grains formed in $\sim 10^{5} \mathrm{y}$. Olivine and anorthite respond differently to solar wind irradiation. The space weathering effects in olivine are particularly difficult to reconcile with laboratory irradiation studies and numerical models. Additional measurements, experiments, and modeling are required to resolve the discrepancies among the observations and calculations involving solar wind amorphization of different minerals on airless bodies.

\section{References:}

[1] LP Keller and S Zhang, LPI Workshop on Space Weathering (2015) p. 2056.

[2] LP Keller and EL Berger, Earth and Planetary Science 66 (2014), p.71.

[3] LP Keller et al., Lunar \& Planetary Science Conference (2016) p. 2525.

[4] EL Berger and LP Keller, Microscopy Today 23 (2015) p.18.

[5] EL Berger and LP Keller, Lunar \& Planetary Science Conference (2015) p. 1543.

[6] GE Blanford et al., Proceedings of the Lunar \& Planetary Science Conference (1975) p. 3557.

[7] T Noguchi et al., Meteoritics and Planetary Science 49 (2014) p.188.

[8] D Harries and F Langenhorst, Earth and Planetary Science 66 (2014), p.163.

[9] EL Berger and LP Keller, Lunar \& Planetary Science Conference (2015) p. 2351.

[10] R Christoffersen and LP Keller, Lunar \& Planetary Science Conference (2015) p. 2084. [11] T Matsumoto et al., LPI Workshop on Space Weathering (2015) p. 2045.

[12] M Lazzarin, The Astrophysical Journal 647 (2006) p. L179.

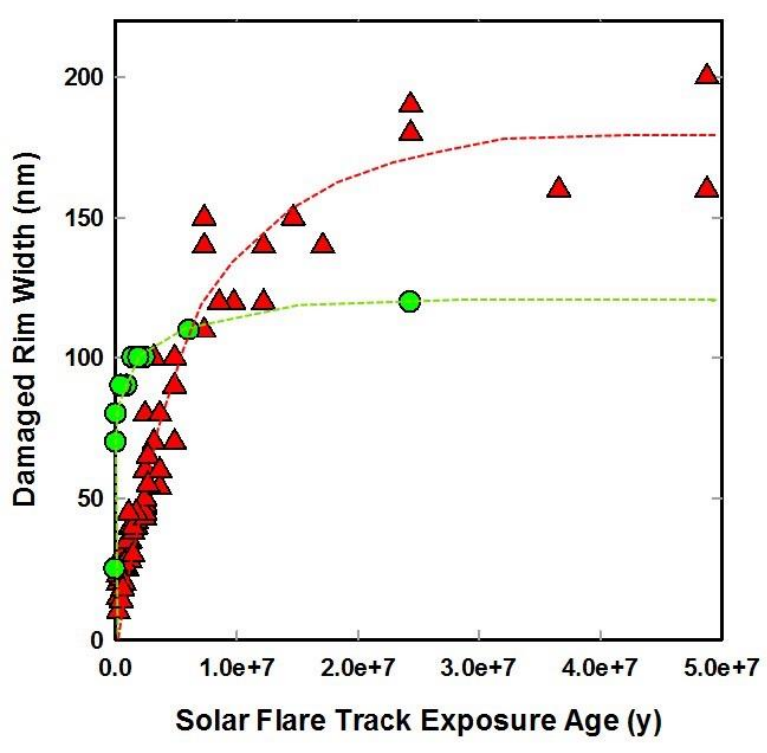

Figure 1. A plot of solar wind damaged rim width vs. solar flare track exposure age for rims on lunar anorthite grains (red triangles) and Itokawa and lunar olivine grains (green circles). The measurement from the olivine in Apollo sample 64455 anchors the curve (100 $\mathrm{nm}$ damaged rim width, 2 My exposure). 